\title{
O papel do divertimento e das determinantes motivacionais na persistência da prática de exercício físico
}

\author{
The role of enjoyment and motivational determinants \\ in persistence in the practice of physical exercise
}

Filipe Rodrigues (https://orcid.org/0000-0003-1327-8872) ${ }^{1}$

Diogo Teixeira (https://orcid.org/0000-0003-4587-5903) ${ }^{2}$

Rita Macedo (https://orcid.org/0000-0001-5861-4089) ${ }^{3}$

Henrique Neiva (https://orcid.org/0000-0001-9283-312X) ${ }^{4}$

Luís Cid (https://orcid.org/0000-0001-8156-3291) ${ }^{1}$

Diogo Monteiro (https://orcid.org/0000-0002-7179-6814) ${ }^{5}$

${ }^{1}$ Escola Superior de Desporto de Rio Maior. Av. Dr. Mário Soares 110. 4020-413 Rio Maior Portugal.frodrigues@ esdrm.ipsantarem.com ${ }^{2}$ Universidade Lusófona.

Lisboa Portugal.

${ }^{3}$ Centro de Estudos

Organizacionais e Sociais do

Politécnico do Porto. Porto

Portugal.

${ }^{4}$ Universidade da Beira

Interior. Covilhã Portugal.

${ }^{5}$ Politécnico de Leiria. Leiria

Portugal.

\begin{abstract}
The scope of this paper was to analyze the impact of motivational determinants in enjoyment and persistence in physical exercise among practitioners of physical exercise. In total, 967 gym and health club exercise practitioners aged between 18 and $65(M=45.08 ; S D=13.76)$ were recruited for analysis. All participants had more than six months of regular exercise practice. Participants completed and validated scales in the exercise context duly translated into Portuguese, assessing interpersonal behaviors, basic psychological needs, and behavioral regulation. Persistence was measured using computerized records considering persistent exercisers as being those who were exercising at similar frequencies as those self-reported at the initial assessment. Results showed that the measurement and structural model fit the data. Several significant effects were found supporting previous literature. Indirect effects showed enjoyment to play a crucial role on exercise persistence, both by the significant effect via autonomous and controlled motivation. In essence, activities that give pleasure that gym and health club exercisers experience during the practice of physical exercise can be the key variable of long-term persistence.
\end{abstract}

Key words Motivation, Enjoyment, Physical exercise
Resumo Como objetivo deste trabalho, definiuse analisar os efeitos indiretos de determinantes motivacionais no divertimento e na persistência em praticantes de exercício físico regular. Participaram ao todo 967 praticantes de exercício em ginásio e health clubs, com idades compreendidas entre 18 e 65 anos $(M=45,08 ; D P=13,76)$. Todos os participantes tinham mais de 6 meses de experiência regular em exercício fisico. Os participantes preencheram ao todo questionários traduzidos e validados para a língua portuguesa no contexto do exercício físico, que examinavam a perceção dos comportamentos interpessoais, as necessidades psicológicas básicas, a regulação da motivação e o divertimento. A persistência foi medida através de registos eletrónicos, considerando um praticante persistente aquele que tivesse uma frequência semanal similar aquela auto-reportada no momento inicial. A maioria das regressões são significativas, confirmado os pressupostos da literatura existente. Os efeitos indiretos mostram que o divertimento desempenha um papel crucial na persistência, tanto por via motivação autónoma, como por via motivação controlada. Em suma, atividades promotoras do prazer que os praticantes de ginásio e health club experienciam durante a prática de exercício físico poderão ser a variável chave na persistência a longo prazo.

Palavras-chave Motivação, Divertimento, Exercício físico 


\section{Introdução}

Empiricamente, pareceexistir uma relação entre as nossas emoções e o nosso comportamento. Assume-se que tendemos a procurar atividades nas quais sentimos prazer e bem-estar, evitando aquelas das quais desgostamos. Nesse sentido, quando as pessoas sentem prazer durante ou após uma sessão de treino, é expectável que ela venha a repetir esse comportamento no futuro ${ }^{1}$. Apesar de existirem indícios desta aparente relação, o divertimento tem sido ignorado como um possível determinante da prática de exercício ${ }^{2}$. Aliás, Rhodes et al. ${ }^{3,4}$ revelam que os mecanismos subjacentes ao divertimento na adesão ao exercício físico continuam por explorar.

Uma razão para esta limitação existente na literatura poderá estar relacionada com as abordagens sociocognitivas que têm dominado a investigação, isto é, estudos recentes têm-se focado na análise comportamental, ignorando a avaliação do estado emocional inerente ao ser humano quando realiza um comportamento prazeroso ${ }^{5}$. No entanto, tal como descrito por Rodrigues et al. ${ }^{6}$, a compreensão dos antecedentes responsáveis pelos níveis de divertimento e como este pode estar associado à persistência da prática de exercício físico necessita de ser analisada. Considerando que a motivação tem sido apontada como um dos motivos principais pela prática (ou não) de exercício físico nos relatórios internacionais mais proeminentes ${ }^{7-9}$, parece ser teoricamente possível analisar a sua contribuição como preditor da resposta emocional (e.g., divertimento) subjacente à prática de exercício físico ao longo do tempo.

\section{Modelo teórico}

A Teoria da Autodeterminação, o modelo motivacional mais estudado no contexto do exercício físico ${ }^{6}$, pressupõe que o ser humano tem uma tendência natural para agir em conformidade com o seu estado motivacional num determinado contexto. Alinhado com esta estrutura teórica da Teoria da Autodeterminação (TAD), Vallerand ${ }^{10}$ desenvolveu um modelo sequencial com dois lados, tendo cada um deles implicações significativas no comportamento do indivíduo. Isto é, existe uma sequência teórica mais favorável em resposta a resultados positivos como maiores níveis de divertimento ${ }^{11,12}$, intenção ${ }^{13}$ e adesão à prática de exercício físico ${ }^{14}$. A outra sequência pressupõem um lado mais adverso, sendo positivamente relacionada com consequências nega- tivas como o esgotamento físico ${ }^{15}$, mau $\operatorname{estar}^{16}$ e desistência da prática de atividade física ${ }^{17}$.

No contexto em análise, o praticante de exercício físico que percecione um técnico de exercício como uma figura de suporte, que criam condições para os indivíduos de regularem o seu próprio comportamento, demonstrando competência, apoio e feedback positivo, está mais próximo de satisfazer as suas NPB, que por sua vez resultará numa regulação do comportamento de forma mais autónoma, ou seja, identifica e integra o comportamento como fazendo parte de $\mathrm{si}^{6}$. No entanto, a regulação do comportamento pode ocorrer de forma oposta. De facto, um praticante que percecione comportamentos interpessoais de frustração (e de certa forma não satisfação), isto é, que, por parte do instrutor identifique comportamentos negligentes, seja alvo de feedback negativo ou sinta pressão imposta, pode estar a regular o seu comportamento de forma controlada, ou seja, envolvido por pressões internas e externas, através da frustração das $\mathrm{NPB}^{18}$.

$\mathrm{O}$ divertimento tem vindo a ser considerado como a variável chave na promoção e manutenção da atividade física ${ }^{1}$, encontrando-se positivamente correlacionado com a motivação autónoma e negativamente com a motivação controlada. A literatura sugere que quanto maior a perceção de prazer e de divertimento, maior será a intenção de realizar esse mesmo comportamento no futuro $^{19}$. Tomando em consideração o divertimento como papel determinante na ligação entre os fatores motivacionais e o comportamento, investigações recentes em contextos similares ao exercício físico têm demonstrado que a motivação autónoma se encontra positivamente associada ao divertimento ${ }^{11}$. Pelo contrário, maiores níveis de motivação controlada têm sido identificados como preditores negativos de sensações de prazer durante a prática de atividade física ${ }^{20}$.

\section{Presente estudo}

Apesar de existirem indícios importantes do papel chave que o divertimento desempenha na manutenção do comportamento, estudos prévios apresentam diversas limitações: I) de acordo com Rodrigues et al. ${ }^{6}$, são escassos os estudos que tenham examinado as duas sequências motivacionais (i.e., mais favorável e mais adversa) como preditoras de um resultado emocional, cognitivo ou comportamental; II) são poucos os estudos que tenham analisado a persistência do comportamento de forma observável ${ }^{14,21}$; III) são escassos os estudos que tenham medido o com- 
portamento (a maioria da literatura apenas recolheu indicadores da intenção de vir a realizar o comportamento $)^{11}$, algo que não se transmite na realização do comportamento per $\mathrm{se}^{13}$; IV) alguns estudos ${ }^{11,22}$ analisaram o papel do clima motivacional, constructo teórico adjacente a outro modelo teórico ${ }^{23}$, sendo limitado a interpretação dos resultados, tal como descrito por Rocchi et al. $^{24} ; \mathrm{V}$ ) estudos existentes têm-se limitado a compreender a associação entre a regulação motivacional e o divertimento, não considerando o seu papel preditor com o comportamento ${ }^{2,25}$.

Assim, o objetivo deste estudo consistiu em analisar os efeitos indiretos de determinantes motivacionais propostos pela teoria da autodeterminação no divertimento e persistência em praticantes de exercício físico regular. De acordo com a literatura existente, especula-se que: i) o divertimento terá um valor preditivo significativo com a persistência ${ }^{26}$; ii) os comportamentos interpessoais de suporte, a satisfação das NPB e a motivação autónoma terão um efeito indireto significativo na persistência via divertimento ${ }^{6}$; e iii) os comportamentos interpessoais de frustração, a frustração das NPB e a motivação controlada terão um efeito indireto significativo negativo, indicando menor persistência ao longo do tempo $^{18}$.

\section{Método}

\section{Participantes}

Uma amostra de conveniência de 967 praticantes de exercício em ginásios e health clubs ( sexo feminino $=778$ ), com idades compreendidas entre 18 e 65 anos $(M=45,08 ; \mathrm{DP}=13,76)$ foram recrutados para participarem neste estudo. Todos os participantes tinham mais de 6 meses de experiência regular em exercício físico $(\mathrm{M}=60,45 ; \mathrm{DP}=9,47)$ e estavam predominantemente envolvidos em aulas de grupo $(\mathrm{N}=444)$, atividades cardiofitness $(\mathrm{N}=378)$ ou em treino personalizado $(\mathrm{N}=145)$. A frequência semanal rondou entre 2 e 7 sessões $(\mathrm{M}=3,21 ; \mathrm{DP}=1,76)$.

\section{Procedimentos}

Todo o processo de recolha dos dados esteve de acordo com a Declaração de Helsínquia e as alterações consequentes. O comité de ética da Universidade da Beira Interior analisou e aprovou o presente estudo. Seguidamente, diversos gestores de ginásios/health clubs foram contac- tados de forma intencional e por conveniência. Os objetivos do estudo foram explicados aos gestores e aprovação foi garantida, dando a autorização aos investigadores de abordarem os praticantes dos ginásios/health clubs para participarem voluntariamente no estudo. Na fase seguinte, potenciais participantes para este estudo foram contactados individualmente na área da receção, previamente ao treino destes. Foi explicado a todos os participantes os objetivos do estudo e foilhes providenciada a informação de anonimato. Todos os participantes que se disponibilizaram a participar voluntariamente neste estudo assinaram consentimento informado previamente ao preenchimento dos questionários.

\section{Instrumentos}

Todos os participantes preencheram quatro questionários traduzidos e validados para a língua portuguesa no contexto do exercício físico. Nomeadamente:

a) O Interpersonal Behavior Questionnaire in Exercise ${ }^{24}$ versão Portuguesa ${ }^{27}$ foi usado para medir a perceção que os praticantes de exercício físico têm sobre os profissionais de exercício com os quais interagem mais durante o treino. Este questionário é composto por 24-itens (quatro itens por fator) que examina os comportamentos de suporte e os comportamentos de frustração. Os participantes respondem a cada item através de uma escala tipo Likert com pontuação mínima 1 (Não concordo de todo) e máximo 7 (concordo totalmente). A versão portuguesa validada por Rodrigues et al. ${ }^{27}$ apresenta valores de Fiabilidade Compósita (FC) acima do valor de corte $(\mathrm{CF}=0,70-0,76)$.

b) O Basic Psychological Need Satisfaction and Frustration Scale ${ }^{28}$ validado no contexto do exercício físico na população portuguesa ${ }^{29}$ foi usado para analisar o grau de satisfação e de frustração das NPB durante a prática de exercício físico. Este instrumento é composto por 24 itens (quatro por fator), aos quais os participantes responderam o seu grau de satisfação de satisfação das três NPB, bem como a sua frustração. Cada item era precedido pela afirmação "eu pratico exercício porque..." qual os participantes responderam usando uma escala tipo Likert com pontuação mínima 1 (discordo totalmente) e máximo 5 (concordo totalmente). Estudos prévios usando este instrumento apresentam valores de consistência interna de acordo com o recomendado ${ }^{30,31}$.

c) O Behavioral Regulation in Exercise Questionnaire $^{32}$ versão Portuguesa no contexto do 
exercício físico ${ }^{33}$ foi usado para medir a forma como os participantes regulam a sua motivação durante a prática de exercício físico. Esta escala composta por 18 itens (três por fator) avalia a perceção que o praticante tem relativamente à forma como cada uma das regulações adjacentes à TAD. Tomando em consideração pressupostos teóricos adjacentes à $\mathrm{TAD}^{34}$, a motivação controlada será composta pela motivação, a regulação externa e a introjetada; a autónoma será composta pela motivação intrínseca, a regulação identificada e integrada. Os participantes respondem a cada item através de uma escala tipo Likert com pontuação mínima 0 (discordo totalmente) e máximo 4 (concordo totalmente). Diversos estu$\operatorname{dos}^{29,30,33}$ têm evidenciado valores de ajustamento aceitáveis, bem como valores de fiabilidade compósita acima dos valores de corte $(>0,70)$.

d) O Physical Activity Enjoyment Scale ${ }^{35}$ traduzido para a língua portuguesa e validado no contexto do exercício físico ${ }^{36}$ foi usado para medir a perceção de divertimento durante a prática de exercício físico. Esta escala é composta por 8 itens que avalia o grau de concordância relativamente à sensação de divertimento durante a prática. Cada item era precedido pela afirmação "Como é que se sente acerca do exercício físico que realiza?" qual os participantes responderam usando uma escala tipo Likert com pontuação mínima 1 (discordo totalmente) e máximo 7 (concordo totalmente). Os autores originais da escala e os autores que fizeram a validade da escala para a língua Portuguesa demonstram valores de ajustamento aceitáveis do modelo, bem como valores de consistência interna aceitáveis.

A persistência foi medida através de registos eletrónicos, dicotomizado o comportamento observável como 1 (persistente) e 0 (desistente). Tomando em consideração a frequência semanal, os participantes eram codificados com o número "1" caso a sua frequência semanal fosse similar durante os seis meses seguintes aquela auto-reportada no momento de avaliação inicial. Participantes que tivessem desistido, abandonado a prática ou tivessem uma frequência semanal significativamente distinta durante os seis meses seguintes ao momento inicial, seriam codificados com o número " 0 ". Estudos prévios analisaram a persistência de forma semelhante ${ }^{21,37}$, demonstrando a fiabilidade de medir o comportamento observável através de um sistema dicotomizado.

\section{Análise estatística}

\section{Análise preliminar}

Dados de questionários com valores omissos $\leq 5 \%$ foram calculados e introduzidos usando o método de Imputação Múltipla descrito por Allison ${ }^{38}$ usando o IBM SPSS Statistics v23. Participantes foram excluídos da análise caso as observações omissas ultrapassassem os $5 \%$. Possíveis outliers univariados $(z>3,00)$ ou multivariados $(\mathrm{D} 2=\mathrm{p} 1<.001, \mathrm{p} 2<.001)$ foram igualmente excluídos da análise ${ }^{39}$. Seguidamente, foram calculados os valores da tendência central e de dispersão, bem como os valores de assimetria e curtose. Para aceitação de uma distribuição normal univariada, os valores de assimetria e de curtose devem estar contidos entre $-2 /+2$ e $-7 /+7$, respetivamente ${ }^{40}$.

Os coeficientes de fiabilidade compósita foram calculados usando a formula de Raykov ${ }^{41}$ para examinar se os itens se propõem a medir o fator latente, assumindo valores $\geq 0,70$ como aceitáveis $^{42}$. Foram calculadas as correlações bivariadas de Pearson considerando correlação fraca $r=0,15$; forte $r=0,60$ e perfeita $r=1$, tal como preconizado por Cohen ${ }^{43}$. Correlações parciais, controlando para o divertimento e a frequência total durante os seis meses seguintes à avaliação inicial.

\section{Modelo de medida e modelo de equações estruturais}

Foi realizada um modelo de medida através de uma Análise Fatorial Confirmatória (AFC) e um modelo de Equações Estruturais (EE) de acordo com as recomendações de $\mathrm{Kline}^{44}$. O modelo de medida e de EE foram analisados no programa Mplus $7.3^{45}$ usando o estimador Robusto da Máxima Verosimilhança dado ser robusto contra a não normalidade da distribuição da amostra. O teste do qui quadrado (2) e os seus respetivos graus de liberdade ( $\mathrm{gl}$ ) não serão analisados nem tomando em consideração para aceitação do modelo, dado ser influenciado pelo tamanho da amostra e pela complexidade do modelo ${ }^{46}$. Nesse sentido, serão tomados em consideração os valores de ajustamento tradicionais e incrementais descritos na literatura ${ }^{46,47}$, nomeadamente: Comparative Fit Index (CFI), Tucker -Lewis Index (TLI), Standard Root Mean Residual (SRMR), Root Mean Square Error of Approximation (RMSEA) e o seu respetivo Intervalo de Confiança a 90\% (IC90\%). Para os referidos indices foram adotados os seguintes valores de corte: CFI e TLI $\geq 0,90$, SRMR e RMSEA $\leq 0,8^{46,47}$. 
or preditivo do divertimento na persistência dos praticantes, como a análise dos efeitos indiretos das determinantes motivacionais na persistência, via o fator divertimento. A significância dos coeficientes estandardizados foi medida através do Intervalo de Confiança (IC) a 95\%, considerando significativo caso este não englobasse o valor de $0^{48}$.

Tomando em consideração o número de parâmetros a serem estimados (i.e., 72 parâmetros), este estudo cumpre com o rácio mínimo de cinco participantes por parâmetro, tal como preconizado pela literatura ${ }^{46,49}$. Como análise complementar relativamente ao tamanho da amostra, foi usado o software $G^{\star}$ Power $3.1^{50}$ para calcular o número mínimo de participantes. Tomando em consideração o número de regressões $(n=9)$ e o effect size antecipado def $2=.01, \alpha=.05$, e poder estatístico de $=.95$, o software sugere uma amostra mínima de 245 participantes, tamanho este que foi respeitado no presente estudo.

\section{Resultados}

\section{Resultados preliminares}

Os dados foram imputados em 25 participantes, dado apresentarem valores omissos em menos de $5 \%$ nos seus questionários. Não foi encontrado nenhum outlier univariado, no entanto, 13 participantes não foram considerados para análise dado apresentarem-se como outliers multivariados.

Na Tabela 1, é possível de observar que a perceção de comportamentos interpessoais de suporte, a satisfação das NPB e a motivação autónoma apresentam valores médios superiores comparativamente à perceção de comportamentos interpessoais de frustração, a frustração das NPB e a motivação controlada. O divertimento apresenta a média mais alta comparativamente às determinantes motivacionais. De acordo com registros eletrônicos, 572 participantes (59\%) mantiveram uma prática de exercício físico regular similar $(\mathrm{M}=3,09 ; \mathrm{DP}=1,45)$ durante os 6 meses após aquela prática auto-reportada na avaliação inicial. Pelo contrário, 251 participantes no presente estudo foram codificados com desistentes, dado terem abandonado a prática nos primeiros dois a quatro meses $(n=113)$ ou apresentarem uma prática de exercício físico regular significativamente diferente $(\mathrm{n}=138)$ aquela prática auto-reportada na avaliação inicial.

Todas as variáveis apresentaram uma distribuição normal, uma vez que os valores de assimetria e de curtose estarem contidos dentro dos valores de corte. Os coeficientes de fiabilidade compósita apresentam valores $>0,70$, apresentado consistência interna. Todas as correlações apresentaram valores significativos, tal como hipoteticamente descrito na literatura: i) os comportamentos interpessoais de suporte, a satisfação das NPB, a motivação autónoma, o divertimento e a persistência estão positivamente correlacionados entre eles; os comportamentos interpessoais de frustração, a frustração das NPB e a motivação controlada estão positivamente correlacionados entre eles, e negativamente com as restantes determinantes motivacionais, com o divertimento e a persistência. Os valores das correlações parciais, controlando para o divertimento e a frequência total ao mesmo tempo foram similares aos obtidos nas correlações de Pearson. Aquando do controlo pelo divertimento e a frequência das sessões de treino, os resultados mostraram coeficientes similares aos obtidos nas correlações bivariadas. Para mais informações ver Tabela 1.

Os valores de VEM apresentam valores acima dos propostos pela literatura. Olhando para o quadrado das correlações, é possível de observar que o valor é inferior aos valores obtidos na análise do VEM. Nesse sentido, pode-se afirmar que foi alcançada a validade convergente e discriminante, respetivamente.

\section{Modelo de medida e modelo de equações estruturais}

Na Tabela 2 é possível observar os valores de ajustamento tradicionais e incrementais para ambos os modelos realizados. Os indicadores mostram que os modelos de medida, resultante da AFC e o modelo de EE obtiveram valores de ajustamento aceitáveis.

$\mathrm{Na}$ Figura 1 está representado o modelo hipotético proposto, bem como os valores das regressões entre os diversos construtos. De acordo com a Figura 1, é possível de evidenciar que a quase totalidade das regressões são significativas 
Tabela 1. Análise da tendência central e de dispersão, distribuição da normalidade, fiabilidade compósita, variância extraída média e correlações entre as variáveis em estudo.

\begin{tabular}{|c|c|c|c|c|c|c|c|c|c|c|c|c|c|c|}
\hline & \multirow{2}{*}{$\mathbf{M}$} & \multirow{2}{*}{ DP } & \multirow{2}{*}{ A } & \multirow{2}{*}{$\mathrm{C}$} & \multirow{2}{*}{ FC } & \multirow{2}{*}{ VEM } & \multicolumn{8}{|c|}{$\mathbf{r}$} \\
\hline & & & & & & & 1 & 2 & 3 & 4 & 5 & 6 & 7 & 8 \\
\hline 1. CI Suporte & 5,49 & 0,83 & $-0,33$ & 0,51 & 0,82 & 0,78 & 1 & & & & & & & \\
\hline 2. CI Frustração & 1,86 & 0,93 & 1,29 & 2,47 & 0,75 & 0,78 & $\begin{array}{r}-0,50 \\
(0,25)\end{array}$ & 1 & & & & & & \\
\hline $\begin{array}{l}\text { 3. Satisfação } \\
\text { NPB }\end{array}$ & 4,18 & 0,53 & $-0,39$ & 0,76 & 0,72 & 0,69 & $\begin{array}{r}0,51 \\
(0,26)\end{array}$ & $\begin{array}{r}-0,24 \\
(0,06)\end{array}$ & 1 & & & & & \\
\hline $\begin{array}{l}\text { 4. Frustração } \\
\text { NPB }\end{array}$ & 1,84 & 0,66 & $-0,86$ & 1,48 & 0,78 & 0,74 & $\begin{array}{r}-0,35 \\
(0,12)\end{array}$ & $\begin{array}{r}0,46 \\
(0,21)\end{array}$ & $\begin{array}{r}-0,54 \\
(0,29)\end{array}$ & 1 & & & & \\
\hline $\begin{array}{l}\text { 5. Motivação } \\
\text { Autónoma }\end{array}$ & 3,40 & 0,54 & $-0,86$ & 1,40 & 0,70 & 0,74 & $\begin{array}{r}0,40 \\
(0,16)\end{array}$ & $\begin{array}{r}-0,27 \\
(0,07)\end{array}$ & $\begin{array}{r}0,40 \\
(0,16)\end{array}$ & $\begin{array}{r}-0,37 \\
(0,14)\end{array}$ & 1 & & & \\
\hline $\begin{array}{l}\text { 6. Motivação } \\
\text { Controlada }\end{array}$ & 0,54 & 0,51 & 1,27 & 2,57 & 0,79 & 0,67 & $\begin{array}{r}-0,21 \\
(0,04)\end{array}$ & $\begin{array}{r}0,43 \\
(0,18)\end{array}$ & $\begin{array}{r}-0,21 \\
(0,04)\end{array}$ & $\begin{array}{r}0,51 \\
(0,26)\end{array}$ & $\begin{array}{r}-0,36 \\
(0,13)\end{array}$ & 1 & & \\
\hline 7. Divertimento & 5.87 & 0,94 & $-0,53$ & $-0,26$ & 0,94 & 0,82 & $\begin{array}{r}0,43 \\
(0,18)\end{array}$ & $\begin{array}{r}-0,20 \\
(0,04)\end{array}$ & $\begin{array}{r}0,35 \\
(0,12)\end{array}$ & $\begin{array}{r}-0,26 \\
(0,07)\end{array}$ & $\begin{array}{r}0,52 \\
(0,27)\end{array}$ & $\begin{array}{r}-0,14 \\
(0,02)\end{array}$ & 1 & \\
\hline 8. Persistência & - & - & - & - & - & - & $\begin{array}{r}0,50 \\
(0,25) \\
\end{array}$ & $\begin{array}{r}-0,31 \\
(0,09) \\
\end{array}$ & $\begin{array}{r}0,40 \\
(0,16) \\
\end{array}$ & $\begin{array}{r}-0,32 \\
(0,10) \\
\end{array}$ & $\begin{array}{r}0,54 \\
(0,29) \\
\end{array}$ & $\begin{array}{r}-0,27 \\
(0,07) \\
\end{array}$ & $\begin{array}{r}0,81 \\
(0,66) \\
\end{array}$ & 1 \\
\hline
\end{tabular}

M=Média; DP=Desvio Padrão; A=Assimetria; C=Curtose; FC=Fiabilidade Compósita; VEM=Variância Extraída Média; $r=$ correlações; $\mathrm{CI}=$ Comportamentos Interpessoais; NPB=Necessidades Psicológicas Básicas. Valores entre parenteses = quadrado das correlações; todas as correlações são significativas a $\mathrm{p} \leq .01$.

Fonte: Elaborado pelos autores.

Tabela 2. Valores de ajustamento do modelo de medida e do modelo de equações estruturais.

\begin{tabular}{lcccccccc}
\hline & \multirow{2}{*}{$\chi^{2}$} & \multirow{2}{*}{ gl } & CFI & TLI & \multirow{2}{*}{ SRMR } & \multirow{2}{*}{ RMSEA } & \multicolumn{2}{c}{ IC90\% } \\
\hline Modelo AFC & $772,061^{*}$ & 248 & 0,922 & 0,905 & 0,042 & 0,061 & 0,056 & 0,065 \\
Modelo EE & $1057,665^{*}$ & 266 & 0,918 & 0,903 & 0,079 & 0,072 & 0,067 & 0,077 \\
\hline
\end{tabular}

$\chi 2=$ chi-quadrado; gl=graus de liberdade; CFI=Comparative Fit Index; TLI=Tucker-Lewis Index; SRMR=Standard Root Mean Residual; RMSEA=Root Mean Square Error of Approximation; IC90\%=Intervalo de Confiança a 90\%; LI=Limite Inferior; LS=Limite Superior; AFC=Análise Fatorial Confirmatória; EE=Equações Estruturais. ${ }^{*}$ Significativo a $p<.001$.

Fonte: Elaborado pelos autores.

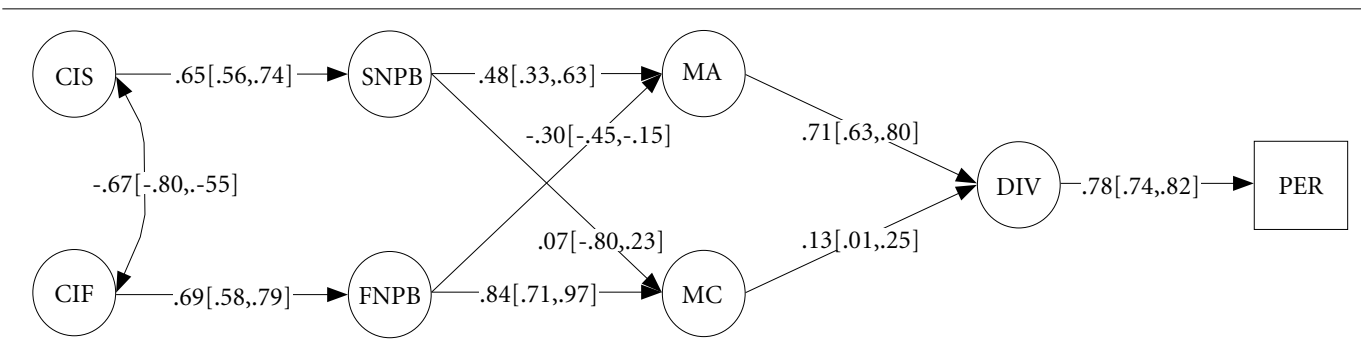

Figura 1. Modelo de equações estruturais.

$\mathrm{CIS} / \mathrm{F}=$ Comportamentos Interpessoais de Suporte; $\mathrm{CIF}=\mathrm{CF}$ de Frustração; $\mathrm{SNPB}=$ Satisfação das Necessidades Psicológicas Básicas; FNPB=Frustração das NPB; MA=Motivação Autónoma; MC=Motivação Controlada; DIV=Divertimento; PER=Persistência; coeficientes estandardizados fora dos parenteses retos; valores dentro dos parenteses retos = coeficientes do limite inferior e superior, tomando em consideração o intervalo de confiança a 95\%.

Fonte: Elaborado pelos autores. 
com IC a 95\%, exceto a SNPB com a motivação controlada. Especificamente, os valores das regressões parecem estar de acordo com as ligações teóricas propostas na literatura: I) a perceção de comportamentos de suporte tem uma predição positiva e significativa com a satisfação das NPB; II) por sua vez, a satisfação das NPB tem uma predição positiva com a motivação autónoma; III) a perceção de comportamentos de frustração tem uma predição positiva e significativa com a frustração das NPB; IV) que por sua vez, a frustração das NPB tem uma predição positiva com a motivação controlada e uma regressão negativa com a motivação autónoma; V) a motivação autónoma e apresenta uma regressão positiva e significativa com o divertimento, ao passo que a motivação controlada apresenta uma regressão negativa e significativa; VI) o divertimento apresenta um valor de predição positivo e significativo na persistência da prática de exercício físico após seis meses.

Os efeitos indiretos estão apresentados na Tabela 3. Os valores dos efeitos a perceção de comportamentos de suporte, a satisfação das SNPB e a motivação autónoma são positivamente significativos na persistência da prática de exercício físico via divertimento. Pelo contrário, a perceção de comportamentos interpessoais de frustração e a frustração NPB apresentam significância negativa com a persistência da prática de exercício físico via motivação autónoma, sendo positiva e significativa via motivação controlada. Ambas as regulações motivacionais são significativas com a persistência, sendo que a regulação autónoma apresenta um coeficiente consideravelmente superior.

\section{Discussão}

O objetivo deste estudo consistiu em analisar os efeitos indiretos de determinantes motivacionais propostos pela teoria da autodeterminação no divertimento e na persistência em praticantes de exercício físico regular a seis meses. De forma a testar o modelo proposto, foram seguidas diversas linhas teóricas ${ }^{34}$ e empíricas ${ }^{12,26,51}$ existentes na literatura. Uma importante implicação deste estudo é que a participação derivada de sensações de prazer pode ser um indicativo significativo na prática continuada de exercício físico a longo prazo.

A TAD assume um papel relevante e contemporâneo, devido aos resultados robustos obtidos em diversos estudos no contexto do exercício físico $^{6,52,53}$. Tal como teoricamente demonstrado ${ }^{34} \mathrm{e}$ empiricamente testado no contexto desporto ${ }^{19,54}$ e na educação física ${ }^{22}$, os resultados deste estudo sugerem que a forma como os praticantes de atividade física percecionam pessoas em posições superiores (e.g., treinadores), tem uma relação forte e significativa na forma como as NPB serão satisfeitas ou frustradas.

A satisfação das NPB mostrou ser um preditor significativo da motivação autónoma, não apresentando qualquer relação com a motivação

Tabela 3. Efeitos indiretos das variáveis motivacionais para a persistência.

\begin{tabular}{|c|c|c|c|}
\hline \multirow{2}{*}{ Caminho } & \multirow{2}{*}{$\boldsymbol{\beta}$} & \multicolumn{2}{|c|}{ CI95\% } \\
\hline & & LI & LS \\
\hline $\mathrm{CI}$ Suporte $\rightarrow \mathrm{SNPB} \rightarrow$ AUTO $\rightarrow$ DIV $\rightarrow$ Persistência & 0,17 & 0,11 & 0,24 \\
\hline CI Suporte $\rightarrow \mathrm{SNPB} \rightarrow \mathrm{CONT} \rightarrow \mathrm{DIV} \rightarrow$ Persistência & 0,02 & 0,00 & 0,03 \\
\hline CI Frustração $\rightarrow$ FNPB $\rightarrow$ AUTO $\rightarrow$ DIV $\rightarrow$ Persistência & $-0,12$ & $-0,17$ & $-0,06$ \\
\hline CI Frustração $\rightarrow$ FNPB $\rightarrow$ CONT $\rightarrow$ DIV $\rightarrow$ Persistência & 0,06 & 0,01 & 0,11 \\
\hline $\mathrm{SNPB} \rightarrow \mathrm{AUTO} \rightarrow$ DIV $\rightarrow$ Persistência & 0,27 & 0,19 & 0,35 \\
\hline $\mathrm{SNPB} \rightarrow \mathrm{CONT} \rightarrow \mathrm{DIV} \rightarrow$ Persistência & 0,02 & 0,01 & 0,03 \\
\hline FNPB $\rightarrow$ AUTO $\rightarrow$ DIV $\rightarrow$ Persistência & $-0,17$ & $-0,24$ & $-0,09$ \\
\hline $\mathrm{FNPB} \rightarrow \mathrm{CONT} \rightarrow \mathrm{DIV} \rightarrow$ Persistência & 0,08 & 0,02 & 0,15 \\
\hline AUTO $\rightarrow$ DIV $\rightarrow$ Persistência & 0,56 & 0,50 & 0,62 \\
\hline CONT $\rightarrow$ DIV $\rightarrow$ Persistência & 0,10 & 0,02 & 0,18 \\
\hline
\end{tabular}

$\beta=$ coeficientes estandardizados; IC95\%=Intervalo de Confiança a 95\%; LI=Limite Inferior; LS=Limite Superior; $\mathrm{CI}=$ Comportamentos Interpessoais; $\mathrm{SNPB}=$ Satisfação das Necessidades Psicológicas Básicas; FNPB=Frustração das NPB; AUTO=Motivação Autónoma; CONT=Motivação Controlada; DIV=Divertimento. 
controlada. Pelo contrário, a frustração das NPB mostrou ser um preditor significativo da motivação controlada e ter uma relação negativa e significativa com a motivação autónoma. Esta sequência corrobora a literatura ${ }^{6,11,30}$ destacando que a forma como a pessoa experiencia a satisfação ou frustração das NPB é crucial na forma como esta irá regular o seu comportamento.

Curiosamente os comportamentos interpessoais de frustração e por sua vez a frustração das NPB predizem negativamente a persistência via motivação autónoma, enquanto conjeturam positivamente via motivação controlada. Acreditase que estes resultados possam estar relacionados com o valor preditivo da frustração das NPB com as regulações motivacionais. Isto é, a regulação autónoma pode estar a diminuir o poder explicativo ao estar negativamente associada com os comportamentos interpessoais de frustração e a frustração das NPB. Por outro lado, a motivação controlada mantém-se positiva e significativa com a frustração das BPN e com o divertimento. Contudo, é de salvaguardar que o poder explicativo dos comportamentos interpessoais de frustração e a frustração das NPB com a persistência é residual via motivação controlada.

Os resultados mostraram que a motivação autónoma teve uma regressão positiva com o divertimento, enquanto que a motivação controlada apresentou um efeito oposto. Esse resultado reforça que a motivação autónoma está tipicamente relacionada a diferentes tipos de resultados positivos, sendo mais favorável a respostas comportamentais positivas com a prática de exercício físico ${ }^{14,52}$. Ou seja, quanto maior for a sensação de divertimento adjacente à prática de exercício físico, maior será a probabilidade de o praticante repetir o comportamento no futuro (61\% de variância explicada no modelo atual).

A motivação autónoma e a controlada têm um efeito indireto na persistência via divertimento. Isto explica que, pessoas que praticam exercício físico porque valorizam o comportamento estão mais predispostas a experienciarem sensações de prazer e, por consequência, mantê -lo no futuro. Estes resultados estão claramente alinhados com os pressupostos da $\mathrm{TAD}^{34}$ e com o modelo proposto por Vallerand ${ }^{10}$, preconizando que a motivação autónoma está positivamente associada a comportamentos e respostas emocionais positivas, apresentando-se como o protótipo do comportamento autodeterminado. Não obstante, a motivação controlada pode predizer o comportamento, via divertimento.

\section{Limitações}

Apesar de este estudo apresentar avanços científicos relevantes para a compreensão do papel chave que o divertimento tem no que diz respeito à persistência da prática de exercício físico, é fundamental perceber as limitações existentes. Em primeiro lugar, deve-se realçar a impossibilidade da generalização dos resultados obtidos para contextos similares. Este estudo é específico à prática de exercício físico em ginásios e health clubs, sugerindo que mais estudos devem ser realizados em outros contextos.

Em segundo lugar, foram calculados fatores compósitos para reduzir o número de parâmetros a serem estimados. Por exemplo, não se sabe o efeito de cada necessidade psicológica básica nas diversas regulações do comportamento. De facto, a complexidade do modelo apresentado demonstra a necessidade de serem feitos mais estudos e com amostras maiores, de forma a garantir um mais concreto entendimento da forma como cada fator impacta a prática de exercício físico.

Por último, o desenho transversal com o follow-up da persistência a seis meses limita a interpretação de inferência, sugerindo que se devem criar intervenções que avaliam as determinantes motivacionais e emocionais. A medição de marcadores fisiológicos que podem estar responsáveis pela realização ou não, da prática de exercício físico, parece ser deveras interessante ${ }^{55}$.

\section{Conclusão}

Os resultados mostram o papel que o divertimento desempenha na persistência da prática de exercício físico. Além disso, este estudo concluiu que os comportamentos interpessoais de suporte percecionados pelos praticantes de ginásio/health clubs predizem indiretamente a persistência, via divertimento. Em suma, os profissionais de exercício e saúde deverão ter em consideração as implicações que os comportamentos interpessoais têm na qualidade da motivação, divertimento e intenção em continuar a prática de exercício, visando a melhoria da participação em contexto de ginásio e atuando mais proximamente na promoção da saúde através do exercício físico.

\section{Implicações práticas}

Programas letivos e formações profissionais na avaliação e prescrição do exercício devem en- 
globar não só, o ensino de conteúdos anatomofisiológicos e biomecânicos, mas igualmente estratégias de mudança comportamental e estudo de teorias psicológicas inerentes ao comportamento humano. Os resultados evidenciam ainda a importância da supervisão e acompanhamento dos técnicos de exercício físico durante a sessão de treino do praticante. Não basta haver a presença de um profissional, é preciso que este adapte os seus comportamentos de acordo com as necessi- dades que os praticantes têm para que seja percecionado como uma figura de suporte. Por último, sugere-se que os técnicos profissionais de saúde e de exercício físico programem e apliquem regularmente atividades lúdicas e sociais, promovendo sensações de satisfação e diversão junto dos praticantes. Tal como descrito pelos resultados, praticantes que experienciem elevados níveis de divertimento têm maiores probabilidades de se manterem na prática após seis meses.

\section{Colaboradores}

F Rodrigues: concebeu este manuscrito e liderou a equipa de redação. D Teixeira: participou na recolha de dados nas análises estatísticas. R Macedo: participou na revisão final e fez contribuições para a seção da discussão. H Neiva: participou na recolha de dados e fez contribuições para a seção da discussão. L Cid: participou na recolha de dados e na revisão do manuscrito. D Monteiro: revisou todo o manuscrito e tomou importantes contribuições em várias seções. Todos os autores leram e aprovaram a versão final do manuscrito.

\section{Financiamento}

Este trabalho foi apoiado pelo Financiamento Nacional através da Fundação para a Ciência e Tecnologia de Portugal, para os autores F Rodrigues, H Neiva, L Cid e D Monteiro. 


\section{Referências}

1. Jekauc D, Brand R. How do Emotions and Feelings Regulate Physical Activity? [editorial]. Front Psychol 2017; 8:1145.

2. Puente R, Anshel M. Exercisers' perceptions of their fitness instructor's interacting style, perceived competence, and autonomy as a function of self-determined regulation to exercise, enjoyment, affect, and exercise frequency. Scand J Psychol 2010; 51:38-45.

3. Rhodes R, McEwan D, Rebar A. Theories of physical activity behaviour change: A history and synthesis of approaches. Psychol Sport Exer 2019; 42:100-109.

4. Rhodes E, Courneya K, Jones L. Translating exercise intentions into behavior: personality and social cognitive correlates. J Health Psych 2003; 8:447-458.

5. Hagger M. Habit and physical activity: Theoretical advances, practical implications, and agenda for future research. Psych Sport Exer 2019; 42:118-129.

6. Rodrigues F, Bento T, Cid L, Neiva HP, Teixeira D, Moutão J, Marinho DA, Monteiro D. Can Interpersonal Behavior Influence the Persistence and Adherence to Physical Exercise Practice in Adults? A Systematic Review. Front Psych 2018; 9:2141.

7. World Health Organisation (WHO). Global Action Plan on Physical Activity 2018-2030. Genebra: WHO; 2019.

8. EC. Special Eurobarometer 472. Sport and Physical Activity. 2018.

9. Physical Activity Council (PAC). 2019 Physical Activity Council's Overview Report on U.S. Participation. Physical Activity Council; 2019.

10. Vallerand R. Toward A Hierarchical Model of Intrinsic and Extrinsic Motivation. Adv Experi Soc Psych 1997; 29:271-360.

11. Monteiro D, Pelletier L, Moutão J, Cid L. Examining the motivational determinants of enjoyment and the intention to continue of persistent competitive swimmers. I J Sport Psych 2018; 49:484-504.

12. Gråstén A, Watt A. A Motivational Model of Physical Education and Links to Enjoyment, Knowledge, Performance, Total Physical Activity and Body Mass Index. J Sports Sci Med 2015; 16:318-327.

13. Hagger $M$, Chatzisarantis $N$. Integrating the theory of planned behaviour and self-determination theory in health behaviour: a meta-analysis. Brit J Health Psych 2009; 14:275-302.

14. Gomes A, Gonçalves A, Maddux J, Carneiro L. The intention-behaviour gap: An empirical examination of an integrative perspective to explain exercise behaviour. I J Sport Exer Psych 2017; 16:607-621.

15. Bartholomew K, Ntoumanis N, Ryan R, Thogersen -Ntoumani C. Psychological need thwarting in the sport context: assessing the darker side of athletic experience. J Sport Exer Psych 2011; 1:75-102.

16. Ng J, Ntoumanis N, Thogersen-Ntoumani C, Stott K, Hindle L. Predicting psychological needs and well -being of individuals engaging in weight management: the role of important others. App Psych Health Well-Being 2013; 5:291-310.

17. Moreno-Murcia J, Huescar Hernandez E, Ruiz L. Perceptions of controlling teaching behaviors and the effects on the motivation and behavior of high school physical education students. Int J Environ Res Public Health 2018; 15(10):2288.
18. Rodrigues F, Teixeira DS, Neiva HP, Cid L, Monteiro D. The bright and dark sides of motivation as predictors of enjoyment, intention, and exercise persistence. Scand J Med Sci Sport 2020; 30(4):787-800.

19. Gardner L, Magee C, Vella S. Social climate profiles in adolescent sports: Associations with enjoyment and intention to continue. J Adolesc 2016; 52:112-123.

20. Navarro-Patón R, Lago-Ballesteros J, Basanta-Camiño $\mathrm{S}$, Arufe-Giraldez V. Relation between motivation and enjoyment in physical education classes in children from 10 to 12 years old. J Hum Sport Exer 2019; 14(3):527-537.

21. Sarrazin P, Vallerand R, Guillet E, Pelletier L, Cury F. Motivation and dropout in female handballers: a 21-month prospective study. Euro J Soc Psych 2002; 32(3):395-418.

22. Gråstén A, Watt A. A motivational model of physical education and links to enjoyment, knowledge, performance, total physical activity and body mas index. $J$ Sport Sci Med 2017; 16(3):318-327.

23. Nicholls JG. Achievement motivation: Conceptions of ability, subjective experience, task choice, and performance. Psycho Rev 1984; 91(3):328-346.

24. Rocchi M, Pelletier L, Cheung S, Baxter D, Beaudry $\mathrm{S}$. Assessing need-supportive and need-thwarting interpersonal behaviours: The Interpersonal Behaviours Questionnaire (IBQ). Personal Indiv Dif 2017; 104:423-433.

25. Pulido J, Sanchez-Oliva D, Amado D, Gonzalez-Ponce I, Sanchez-Miguel P. Influence of motivational processes on enjoymento, boredom and intention to persist in young sportspersons. S Afr J Res Sport Phy Educ Recreat 2014; 36:135-149.

26. Gardner L, Magee C, Vella S. Enjoyment and Behavioral Intention Predict Organized Youth Sport Participation and Dropout. J Physi Acti Health 2017; 14(11):861-865.

27. Rodrigues F, Pelletier L, Neiva H, Teixeira D, Cid L, Monteiro D. Initial validation of the Portuguese version of the Interpersonal Behavior Questionnaire (IBQ \& IBQ-Self) in the context of exercise: Measurement invariance and latent mean differences. Cur Psycho 2021; 40:4040-4051.

28. Chen B, Vansteenkiste M, Beyers W, Boone L, Deci EL, Van der Kaap-Deeder J, Duriez B, Lens W, Matos L, Mouratidis A, Ryan RM, Sheldon KM, Soenens B, Van Petegem S, Verstuyf J. Basic psychological need satisfaction, need frustration, and need strength across four cultures. Mot Emo 2015; 39:216-236.

29. Rodrigues F, Hair J, Neiva H, Teixeira D, Cid L, Monteiro D. The Basic Psychological Need Satisfaction and Frustration Scale in Exercise (BPNSFS-E): Validity, Reliability, and Gender Invariance in Portuguese Exercisers. Perce Motor Skills 2019; 126(5):949-972.

30. Teixeira D, Silva M, Palmeira A. How does frustration make you feel? A motivational analysis in exercise context. Motiv Emo 2018; 42:419-428.

31. Warburton V, Wang J, Bartholomew K, Tuff R, Bishop $K$. Need satisfaction and need frustration as distinct and potentially co-occurring constructs: Need profiles examined in physical education and sport. Motiv Emo 2020; 44:54-66. 
32. Wilson P, Rodgers WT, Rodgers W, Wild C. The Psychological Need Satisfaction in Exercise Scale. $J$ of Sport and Exer Psych. 2006; 28:231-251.

33. Cid L, Monteiro D, Teixeira D, Teques P, Alves S, Moutão J, Silva M, Palmeira A. The Behavioral Regulation in Exercise Questionnaire (BREQ-3) Portuguese-Version: Evidence of Reliability, Validity and Invariance Across Gender. Front Psych 2018; 9:1940.

34. Ryan R, Deci E. Self-determination theory. Basic Psychological Needs in Motivation, Development, and Wellness. New York: Guildford Press; 2017.

35. Mullen SP, Olson EA, Phillips SM, Szabo AN, Wójcicki TR, Mailey EL, Gothe NP, Fanning JT, Kramer AF, McAuley E. Measuring enjoyment of physical activity in older adults: invariance of the physical activity enjoyment scale (paces) across groups and time. Int $J$ Behav Nutr Phys Act 2011; 8:103.

36. Teques P, Calmeiro L, Silva C, Borrego C. Validation and adaptation of the Physical Activity Enjoyment Scale (PACES) in fitness group exercisers. J Sport Health Sci 2020; 9(4):352-357.

37. Gucciardi D, Jackson B. Understanding sport continuation: an integration of the theories of planned behaviour and basic psychological needs. J Sci Med Sport 2015; 18(1):31-36.

38. Allison P. Multiple Imputation for Missing Data: A Cautionary Tale. Sociol Method Res 2000; 28(3):301309.

39. Comrey A. A Method for Removing Outliers to Improve Factor Analytic Results. Multi Behav Res 1985; 20(3):273-281.

40. Gravetter F, Wallnau L. Essentials of Statistics for the Behavioral Sciences. $8^{\text {a }}$ ed. Belmont: Wadsworth Cengage Learning; 2014.

41. Raykov T. Estimation of Composite Reliability for congeneric measures. App Psycho Measurement 1997; 21(2):173-184.

42. Raykov T, Gabler S, Dimitrov D. Maximal Reliability and Composite Reliability: Examining Their Difference for Multicomponent Measuring Instruments Using Latent Variable Modeling. Structural Equation Modeling Multidisc J 2015; 23(3):384-391.

43. Cohen J. Statistical power analysis for the behavioural sciences. $2^{\text {a }}$ ed. New Jersey: Lawrence Earlbaum Associates; 1988.

44. Kline R. Principles and practice of structural equation modelling. $3^{\mathrm{a}}$ ed. New York: The Guildford Press; 2016.

45. Muthén L, Muthén B. Mplus User's Guide. Los Angeles: Muthén \& Muthén; 2010.

46. Hair J, Black W, Babin B, Anderson R. Multivariate Data Analysis. $8^{\text {a }}$ ed. New Jersey: Pearson Educational, Inc; 2019.

47. Byrne B. Structural Equation Modeling with Mplus - Basic Concepts, Applications, and Programming. Abingdon: Routledge; 2011.

48. Williams J, Mackinnon D. Resampling and Distribution of the Product Methods for Testing Indirect Effects in Complex Models. Struct Equ Modeling 2008; 15(1):23-51.
49. Worthington R, Whittaker T. Scale Development Research: A Content Analysis and Recommendations for Best Practices. Counse Psychol 2016; 34(6):806-838.

50. Faul F, Erdfelder E, Buchner A, Lang AG. Statistical power analyses using $G^{*}$ Power 3.1: tests for correlation and regression analyses. Behav Res Methods 2009; 41(4):1149-1160.

51. Gaston A, Jesus S, Markland D, Prapavessis H. I sit because I have fun when I do so! Using self-determination theory to understand sedentary behavior motivation among university students and staff. Health Psychol BehavMed 2016; 4(1):138-154.

52. Teixeira P, Carraca E, Markland D, Silva M, Ryan R. Exercise, physical activity, and self-determination theory: a systematic review. Int J Behav Nutr Phys Act 2012; 9:78

53. Teixeira D, Marques M, Palmeira A. Associations between affect, basic psychological needs and motivation in physical activity contexts: Systematic review and meta-analysis. $R$ Iberoam Psicol Ejerc Depo 2018; 13(2):225-233

54. Teixeira DS, Pelletier LG, Monteiro D, Rodrigues F, Moutão J, Marinho DA, Cid L. Motivational patterns in persistent swimmers: A serial mediation analysis. Euro J Sport Sci 2020; 20(5):660-669.

55. Di Domenico S, Ryan R. The Emerging Neuroscience of Intrinsic Motivation: A New Frontier in Self-Determination Research. Front Hum Neurosci 2017; 11:145.

Artigo apresentado em 25/10/2019

Aprovado em 25/03/2020

Versão final apresentada em 27/03/2020

Editores chefes: Romeu Gomes, Antônio Augusto Moura da Silva 
